\title{
Numerical Estimation of the Effect of the Magnetic Field Application on the Motion of Inclusion in Continuous Casting of Steel
}

\author{
Baokuan LI and Fumitaka TSUKIHASHI ${ }^{11}$ \\ Department of Thermal Engineering, School of Materials and Metallurgy, Northeastern University, Shenyang, 110004, China. \\ 1) Department of Advanced Materials, Graduate School of Frontier Sciences, The University of Tokyo, Kashiwanoha, Kashiwa, \\ Chiba 277-8561 Japan.
}

(Received on October 31, 2002; accepted in final form on April 10, 2003)

\begin{abstract}
Numerical estimation has been conducted to analyze the motion of inclusion considering the effects of argon gas injection and magnetic field application in the continuous casting of slab. The fluid velocity field was obtained by solving the Navier-Stokes equations with electromagnetic force, and the trajectories of inclusion particles are calculated based on the computed velocity field. A reasonable agreement between numerical and experimental trajectory for single sphere was obtained using the water model. The movements of particles are traced in cases with and without the magnetic field and argon gas injection. The results show that some particles after spiral movements re-enter the jet zone of molten steel, and then enter the opposite circulation zone. Inclusions in the upper circulation zone are easily removed. Argon gas injection increases the removal rate of inclusions. The spiral trajectories of inclusion particles disappear when the magnetic field is applied, and the particle velocities decrease significantly. The argon gas injection and magnetic field application are effective for the control of the inclusions.
\end{abstract}

KEY WORDS: continuous casting; inclusion; argon gas injection; magnetic field; computer simulation.

\section{Introduction}

With the development of secondary steelmaking, the technique of removing inclusions has been significantly improved in ladle and tundish, and clean steels have been produced. Nevertheless, some remaining inclusions enter into the molten steel in the casting mold through the submerged entry nozzle. Furthermore, new inclusions are formed due to damage of the nozzle and suction of the molten slag. To improve the steel quality in steelmaking processes, the control of inclusion behavior in continuous casting processes becomes important. As the specific gravity of the inclusion is smaller than that of molten steel, some parts of the inclusions gradually float to the surface of molten steel and are trapped by the slag layer. However, most of the inclusions enter into the casting mold with the flow of molten steel, and cause the defects of products. The problem of removal of inclusions becomes serious when the casting speed is high. Therefore, for improving the removal of inclusions in the mold region of continuous casting, it is important to clarify the behavior of inclusions in the mold.

At high temperature, it is impossible to study directly the motion behavior of inclusions in molten steel in actual continuous casters. Furthermore, it is also hard to maintain the inclusions, similitude in terms of size distribution and physical properties, between the physical model and actual casters. Therefore, it is very difficult to understand the motion behavior of inclusions on the basis of experimental observation and measurement. However, numerical estimation is one of the useful means for understanding the motion behavior of inclusions.

Ho et $a l .{ }^{1)}$ have simulated the trajectories of inclusions in the billet with changing operating conditions of continuous casting. Thomas et al. $^{2,3)}$ examined the behavior of argon gas bubbles and the effect of argon gas bubbles on the inclusion removal in the water model of the continuous casting of slab; it is revealed that the effect of argon gas injection is significant. Taniguchi et al. ${ }^{4)}$ and Miki et al. ${ }^{5)}$ studied the coagulation and diffusion models of inclusion particles in liquid steel. In recent years, more and more systems take advantage of electromagnetic forces to change the flow field in continuous casting processes. ${ }^{6-10)}$ Obviously, an impending problem is the change of the inclusion behavior with the change of flow field affected by magnetic field application. Gardin et al. ${ }^{11)}$ predicted the effect of electromagnetic separation force on the inclusion trajectories. Cho et $a{ }^{12)}$ developed a new EMBR core shape to reduce the inclusion in the fully curved continuous casting machine. Most researchers are in the habit of using the water model to investigate and optimize the inclusion problems, rather than developing a complex cold liquid metal model with mercury or wood's metal. Numerical simulation can provide valuable information at a reasonable cost for the scaling up of the industrial system. In order to evaluate the 


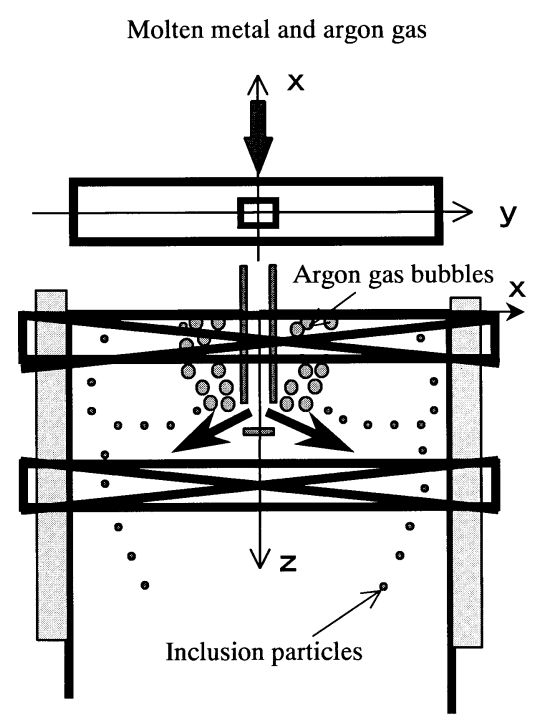

(a)

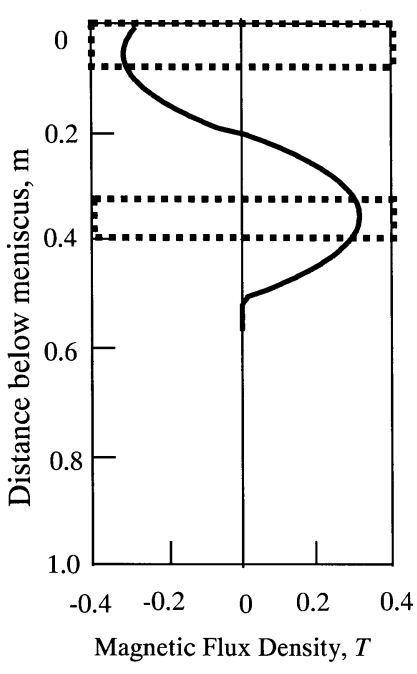

(b)

Fig. 1. Molten metal-gas flow system with the magnets in a continuous casting process. (a) Schematic of physical model, and (b) regression curve of measured magnetic flux density.

cleanliness of molten steel or the inclusions distribution and understand the effect of magnetic field application, a reliable estimation model and method is necessary.

The purposes of the present work are to develop a numerical model of the motion of inclusion considering the effects of the argon gas injection and the magnetic field application, and to understand the removal rates of inclusions by tracing the trajectory of particles.

\section{Mathematical Formulation}

\subsection{Molten Steel Flow}

Figure 1 shows a schematic of the physical model for the molten metal-gas flow system with a magnetic field in a continuous casting mold. To analyze the behavior of inclusion particles, it is necessary to make a model including molten steel flow and the motion of inclusion particles. The flow field of molten metal in the solidifying shell of the strand is assumed to be steady and turbulent. One of the authors $^{13)}$ had studied the flow fields with consideration of the effects of argon gas injection and static magnetic field application in the continuous casting processes; these parameters are shown in Table 1.

\section{(1) Fluid Flow Equations}

The flow behaviors of the molten metal and argon gas are governed by the equations of mass conservation, momentum conservation, and the turbulence model. The equations in the Cartesian coordinate system are written in three-dimensional form as follows.

$$
\begin{aligned}
& \frac{\partial(\rho \phi)}{\partial t}+\frac{\partial(\rho u \phi)}{\partial x}+\frac{\partial(\rho v \phi)}{\partial y}+\frac{\partial(\rho w \phi)}{\partial z} \\
& =\frac{\partial}{\partial x}\left(\Gamma \frac{\partial \phi}{\partial x}\right)+\frac{\partial}{\partial y}\left(\Gamma \frac{\partial \phi}{\partial y}\right)+\frac{\partial}{\partial z}\left(\Gamma \frac{\partial \phi}{\partial z}\right)+S_{\phi}
\end{aligned}
$$

The equations for each variable are shown in Table 2 ,
Table 1. Geometrical parameters and physical properties of steel for simulation.

\begin{tabular}{lc}
\hline Parameters & \multicolumn{2}{c}{ Values } \\
\hline Mold size, $\mathrm{mm}$ & 500 (width) $\times 70$ (thickness) $\times 1000$ (length) \\
Nozzle size, $\mathrm{mm}$ & 34 (thickness) $\times 34$ (width) \\
Depth of nozzle, $\mathrm{mm}$ & 120 \\
Casting speed, $\mathrm{m} / \mathrm{min}$ & 3.5 \\
Inlet gas volume fraction & 0.10 \\
Density of molten steel, $\mathrm{kg} / \mathrm{m}^{3}$ & 7020 \\
Density of inclusion, $\mathrm{kg} / \mathrm{m}^{3}$ & 2780 \\
Viscosity of molten steel, $\mathrm{N} \cdot \mathrm{s} / \mathrm{m}^{2}$ & $5.59 \times 10^{-3}$ \\
Casting temperature, ${ }^{\circ} \mathrm{C}$ & 1550 \\
Electric conductivity of molten steel, $\Omega^{-1} \mathrm{~m}^{-1}$ & $7.14 \times 10^{5}$ \\
Maximum of magnetic flux density, Tesla & 0.315
\end{tabular}

where $\boldsymbol{F}$ is electromagnetic force.

The standard $k-\varepsilon$ two-equation turbulence model ${ }^{13)}$ determines the effective viscosity $\mu_{\mathrm{e}}$.

(2) Effect of Argon Gas Injection

The fluid including molten metal and argon gas is homogeneously modeled by variation of the density of molten metal. $\rho=\rho_{\mathrm{g}} \alpha+(1-\alpha) \rho_{\text {liq, }}$, where $\alpha$ is gas volume fraction, which can be calculated by solving a continuum convection/diffusion equation,

$$
\begin{aligned}
& u \frac{\partial(\rho \alpha)}{\partial x}+v \frac{\partial(\rho \alpha)}{\partial y}+\left(w+w_{\mathrm{s}}\right) \frac{\partial(\rho \alpha)}{\partial z} \\
& =\frac{\partial}{\partial x}\left(\frac{\mu_{\mathrm{e}}}{\sigma_{\alpha}} \frac{\partial \alpha}{\partial x}\right)+\frac{\partial}{\partial y}\left(\frac{\mu_{\mathrm{e}}}{\sigma_{\alpha}} \frac{\partial \alpha}{\partial y}\right)+\frac{\partial}{\partial z}\left(\frac{\mu_{\mathrm{e}}}{\sigma_{\alpha}} \frac{\partial \alpha}{\partial z}\right)
\end{aligned}
$$

where the turbulent Schmidt number $\sigma_{\alpha}$ is set to 1 . To simplify the problem, no momentum equation is solved for the gas phase. However, the bubbles are assumed to reach the 
Table 2. Variables in the common equation.

\begin{tabular}{|c|c|c|c|}
\hline & $\phi$ & $\Gamma$ & $S_{\phi}$ \\
\hline Continuity & 1 & 0 & 0 \\
\hline $\mathrm{x}$-direction momentum & $u$ & $\mu_{\mathrm{e}}$ & $S_{u}=\frac{\partial}{\partial x}\left(\mu_{e} \frac{\partial u}{\partial x}\right)+\frac{\partial}{\partial y}\left(\mu_{e} \frac{\partial v}{\partial x}\right)+\frac{\partial}{\partial z}\left(\mu_{e} \frac{\partial w}{\partial x}\right)-\frac{\partial p}{\partial x}+F_{x}$ \\
\hline y-direction momentum & V & $\mu_{\mathrm{e}}$ & $S_{v}=\frac{\partial}{\partial x}\left(\mu_{e} \frac{\partial u}{\partial y}\right)+\frac{\partial}{\partial y}\left(\mu_{e} \frac{\partial v}{\partial y}\right)+\frac{\partial}{\partial z}\left(\mu_{e} \frac{\partial w}{\partial y}\right)-\frac{\partial p}{\partial y}+F_{y}$ \\
\hline z-direction momentum & W & $\mu_{e}$ & $S_{w}=\frac{\partial}{\partial x}\left(\mu_{e} \frac{\partial u}{\partial z}\right)+\frac{\partial}{\partial y}\left(\mu_{e} \frac{\partial v}{\partial z}\right)+\frac{\partial}{\partial z}\left(\mu_{e} \frac{\partial w}{\partial z}\right)-\frac{\partial p}{\partial z}+F_{z}+\rho g$ \\
\hline $\begin{array}{l}\text { Turbulent kinetic } \\
\text { Energy }\end{array}$ & $k$ & $\frac{\mu_{t}}{\sigma_{k}}$ & $\begin{aligned} S_{k} & =G-\rho \varepsilon \\
G & =\mu_{t}\left\{2\left(\frac{\partial u}{\partial x}\right)^{2}+2\left(\frac{\partial v}{\partial y}\right)^{2}+2\left(\frac{\partial w}{\partial z}\right)^{2}\right. \\
& \left.+\left(\frac{\partial u}{\partial y}+\frac{\partial v}{\partial x}\right)^{2}+\left(\frac{\partial u}{\partial z}+\frac{\partial w}{\partial x}\right)^{2}+\left(\frac{\partial w}{\partial y}+\frac{\partial v}{\partial z}\right)^{2}\right\}\end{aligned}$ \\
\hline $\begin{array}{l}\text { Dissipation rate of } \\
\text { turbulent kinetic energy }\end{array}$ & $\varepsilon$ & $\frac{\mu_{t}}{\sigma_{\varepsilon}}$ & $S_{\varepsilon}=C_{1} G \frac{\varepsilon}{k}-C_{2} \rho \frac{\varepsilon^{2}}{k}$ \\
\hline \multicolumn{4}{|c|}{ Effective viscosity ${ }^{[13]}: \quad \mu_{t}=\frac{C_{\mu} \rho k^{2}}{\varepsilon}, \mu_{\mathrm{e}}=\mu_{\mathrm{L}}+\mu_{t}$} \\
\hline \multicolumn{4}{|c|}{$C_{1}=1.44, C_{2}=1.92, C_{\mu}=0.09, \sigma_{k}=1.0, \sigma_{\varepsilon}=1.3$} \\
\hline
\end{tabular}

steady-state terminal velocity in the vertical direction. The terminal velocity is calculated by the empirical correlation. $^{2)}$

According to previous work, ${ }^{2,3)}$ a reasonable effect of gas bubbles on flow can be obtained when the diameter of the bubbles is set to $3 \mathrm{~mm}$.

(3) Electromagnetic Force

Lorenz's law calculates the electromagnetic force

$$
\boldsymbol{F}=\boldsymbol{J} \times \boldsymbol{B}
$$

where the induced current density $\boldsymbol{J}$ obeys Ohm's law;

$$
\boldsymbol{J}=\sigma(\boldsymbol{E}+\boldsymbol{V} \times \boldsymbol{B})
$$

and $\boldsymbol{E}=-\nabla \varphi$.

The continuity of the induced current density is expressed by the following equation:

$$
\nabla \cdot J=0
$$

hence

$$
\nabla \cdot \sigma \nabla \varphi=\nabla \cdot \sigma(\boldsymbol{V} \times \boldsymbol{B})
$$

$\boldsymbol{B}$ is the magnetic flux density of an imposed magnetic field and is obtained by experiment. The maximum value is $0.315 \mathrm{~T}$ for the mold gap space under a source current of $1000 \mathrm{~A}$ as shown in Fig. 1.

The mushy zone is not included in the domain of calculation to avoid computational difficulties associated with modeling latent heat evolution at the solidification front. The wall function ${ }^{14)}$ is used to calculate the electric potential at a wall boundary while the electric current enters the solidifying shell. A finite volume method is chosen for cal- culation of this complex problem. The nonlinear coupled equations are solved with the SIMPLEC algorithm. The nozzle volume is obtained by employing the blockage technique. The main mesh size is $12 \times 48 \times 82$ for the $x, y$, and $z$ directions, respectively. These results are used to determine the trajectories of inclusion particles.

\subsection{Movement of Inclusions}

In order to analyze the inclusion behavior in a continuous casting process, a mathematical formulation of the three-dimensional motion of particles is applied. Assumptions for the calculation are as follows: (1) The turbulence of the fluid is homogeneous and steady. (2) The particle is spherical and small and its motion does not affect the flow field. (3) Coalescence and break up of inclusion particles are not considered. (4) Argon gas bubbles affect the motion of inclusion particles in the caster by changing the time-averaged flow field and turbulence intensifies. In this study, the entrapment of inclusion has not been taken into account to examine the effect of DC magnetic field and argon gas injection on the behavior of inclusion in the strand pool.

The behavior of inclusion particles is obtained by solving the following equation. ${ }^{15)}$

$$
\rho_{\mathrm{p}} \frac{\pi}{6} d_{\mathrm{p}}^{3} \frac{\boldsymbol{d} \boldsymbol{V}_{\mathrm{p}}}{\boldsymbol{d} \boldsymbol{t}}=\boldsymbol{F}_{\mathrm{g}}+\boldsymbol{F}_{\mathrm{f}}+\boldsymbol{F}_{\mathrm{d}}+\boldsymbol{F}_{\mathrm{A}}+\boldsymbol{F}_{\mathrm{P}}+\boldsymbol{F}_{\mathrm{part}}+\boldsymbol{F}_{\mathrm{h}}
$$

The right-hand side is the force required to accelerate the particle.

$\boldsymbol{F}_{\mathrm{g}}$ is the gravitational force of the inclusion particle;

$$
\boldsymbol{F}_{\mathrm{g}}=\rho_{\mathrm{p}} \frac{\pi}{6} d_{\mathrm{p}}^{3} \boldsymbol{g}
$$


$\boldsymbol{F}_{\mathrm{f}}$ is the buoyancy force acting on the inclusion particle;

$$
\boldsymbol{F}_{\mathrm{f}}=\rho_{1} \frac{\pi}{6} d_{\mathrm{p}}^{3} \boldsymbol{g}
$$

$\boldsymbol{F}_{\mathrm{d}}$ is the viscous drag force;

$$
\boldsymbol{F}_{\mathrm{d}}=\frac{\pi}{8} C_{\mathrm{d}} \rho_{\mathrm{l}} d_{\mathrm{p}}^{2}\left(\boldsymbol{V}_{\mathrm{p}}-\boldsymbol{V}\right)\left|\boldsymbol{V}_{\mathrm{p}}-\boldsymbol{V}\right|
$$

where $\boldsymbol{V}_{\mathrm{p}}$ and $\boldsymbol{V}$ represent the instantaneous velocity of the particle and the undisturbed fluid velocity at the center of the particle. $C_{\mathrm{d}}$ represents the drag coefficient, ${ }^{16)}$

$$
\begin{array}{ll}
C_{\mathrm{d}}=\frac{24}{\operatorname{Re}}\left(1+0.15 \mathrm{Re}^{0.678}\right), & \mathrm{Re} \leq 1000 \\
C_{\mathrm{d}}=0.44, & \mathrm{Re}>1000
\end{array}
$$

where

$$
\operatorname{Re}=\frac{\rho d_{\mathrm{p}}\left|\boldsymbol{V}_{\mathrm{p}}-\boldsymbol{V}\right|}{\mu_{1}}
$$

$\boldsymbol{F}_{\mathrm{A}}$ is the added mass force.

The added mass force takes into account the fact that an accelerating or decelerating body must also accelerate a volume of surrounding liquid equal to one-half of the volume a sphere displaces,

$$
\boldsymbol{F}_{\mathrm{A}}=\rho_{1} C_{\mathrm{A}} \frac{\pi}{6} d_{\mathrm{p}}^{3}\left(\frac{\boldsymbol{D} \boldsymbol{V}}{\boldsymbol{d} \boldsymbol{t}}-\frac{\boldsymbol{d} \boldsymbol{V}_{\mathrm{p}}}{\boldsymbol{d} \boldsymbol{t}}\right)
$$

where $C_{\mathrm{A}}=0.5$.

$\boldsymbol{F}_{\mathrm{P}}$ is due to the pressure gradient in the fluid surrounding the particle caused by acceleration of fluid,

$$
\boldsymbol{F}_{\mathrm{P}}=\rho_{\mathrm{p}} \frac{\pi}{6} d_{\mathrm{p}}^{3} \frac{\boldsymbol{D} \boldsymbol{V}}{\boldsymbol{d} \boldsymbol{t}}
$$

The inclusions present in the molten steel experience a force in the direction opposite to that of the applied electromagnetic force. This force acting on the inclusions is called the Archimedes electromagnetic force. ${ }^{17)}$ As electrical conductivities of inclusion and liquid steel are different, the force is derived by the following formulation.

$$
\boldsymbol{F}_{\text {part }}=-\psi(\boldsymbol{J} \times \boldsymbol{B}) \text {. }
$$

where $\psi$ is a form factor.

If inclusions are spherical non-conducting particles, the factor is $\psi=(3 / 4)(\pi / 6) d_{\mathrm{p}}^{3}$.

In the present problem, the electromagnetic separation force is added; its direction is coincident with velocities of molten steel but opposite to the Lorentz force. To estimate the available magnitude of the electromagnetic force, let us consider a current density of $1.0 \times 10^{4} \mathrm{~A} / \mathrm{m}^{2}{ }^{2}$ ) If $\boldsymbol{B}$ is approximately 0.3 Tesla, for an inclusion particle, the electromagnetic force value is nearly 0.1 times the gravitational force of inclusions. If the inclusion particle is in the flow field with the large velocity gradient, it is necessary to consider the effect of the electromagnetic separation force.

The Basset history force, $\boldsymbol{F}_{\mathrm{h}}$, takes into account the development of vortices around the accelerating inclusion.

$$
\boldsymbol{F}_{\mathrm{h}}=6 a^{2}\left(\pi \mu \rho_{\mathrm{f}}\right)^{1 / 2} \int_{0}^{t} \frac{\boldsymbol{d}\left(\boldsymbol{V}-\boldsymbol{V}_{\mathrm{p}}\right) / \boldsymbol{d} \boldsymbol{\tau}}{(\boldsymbol{t}-\boldsymbol{\tau})^{1 / 2}} d \boldsymbol{\tau}
$$

Gardin et al. ${ }^{11)}$ found that the history term can be neglected when the diameter of particles is smaller than 620 $\mu \mathrm{m}$. The present model neglects this term, since the focus is on the inclusion particles in molten steel with diameters less than $200 \mu \mathrm{m}$.

Total derivation should be considered: $\boldsymbol{D} \boldsymbol{V} / \boldsymbol{d} \boldsymbol{t}=\partial \boldsymbol{V} / \partial \boldsymbol{t}+$ $\boldsymbol{V} \cdot \boldsymbol{\nabla} \boldsymbol{V}$, and is $\boldsymbol{D V} / \boldsymbol{d} \boldsymbol{t}=\boldsymbol{V} \cdot \boldsymbol{\nabla} \boldsymbol{V}$ for the constant flow.

Particle location is obtained by:

$$
\frac{d x_{\mathrm{p}}}{d t}=V_{\mathrm{p}}
$$

where $\boldsymbol{x}_{\mathrm{p}}$ is the particle coordinate vector.

The Runge-Kutta-Gill method is used to solve the motion equations. Time step is set to $0.001 \mathrm{~s}$, the tracing time is $60 \mathrm{~s}$, and a slab of $3.5 \mathrm{~m}$ lengths can be cast out during this time. The number of inclusions traced during $60 \mathrm{~s}$ was varied from 1 to 400 .

The initial condition of inclusion particles includes initial location and velocities. The initial locations are uniformly distributed within the horizontal section of the nozzle, since the uniform distribution of inclusions is assumed. The initial velocities of particles are local velocities of fluid that are obtained by geometrical interpolation.

\section{Results and Discussion}

\subsection{Experimental Verification for Single Sphere Par- ticles}

In order to verify the numerical model, a water model experiment is carried out. The transparent vessel is made of plexiglas and the size of its section is same as that of the actual mold section in Table 1. Water was used as modeling fluid with satisfying that the Reynolds and Froude numbers are approximately same at given volumetric throughput in both systems. A brake handle valve was used to control the flow rate from $1.73 \times 10^{-3} \mathrm{~m}^{3} / \mathrm{s}$ to $2.16 \times 10^{-3} \mathrm{~m}^{3} / \mathrm{s}$ that correspond to casting speed from 3 to $4 \mathrm{~m} / \mathrm{min}$. The flow passage is snake-shaped so as to shorten the length of the vessel as shown in Fig. 2. Figure 3 shows the computed flow field in the upper part of the water model of continuous casting process. Comparison of flow fields between numerical calculation and water model observation ${ }^{18)}$ has been performed. If the effect of magnetic field application is not considered, the time-averaged flow structures in the mold are analogous even at different liquid densities and viscosities. A plastic sphere with the diameter of $3 \mathrm{~mm}$ is injected into the nozzle from the upper water pool. The density of sphere is $900 \mathrm{~kg} / \mathrm{m}^{3}$. A videotape recorder is used to record the location of the sphere from horizontal and vertical directions. At the same time, the trajectory of sphere without the gas injection and magnetic field application is also simulated. Figure 2 shows the comparison of sphere location obtained by observation and computation. The difference in results may be due to neglect of the rotation of sphere in the mathematical model, since the high rotational speed of the sphere is observed in experiment.

\subsection{Dependence of Particle Number}

Parameters for calculation such as geometry, physical properties, and operating conditions are summarized in 

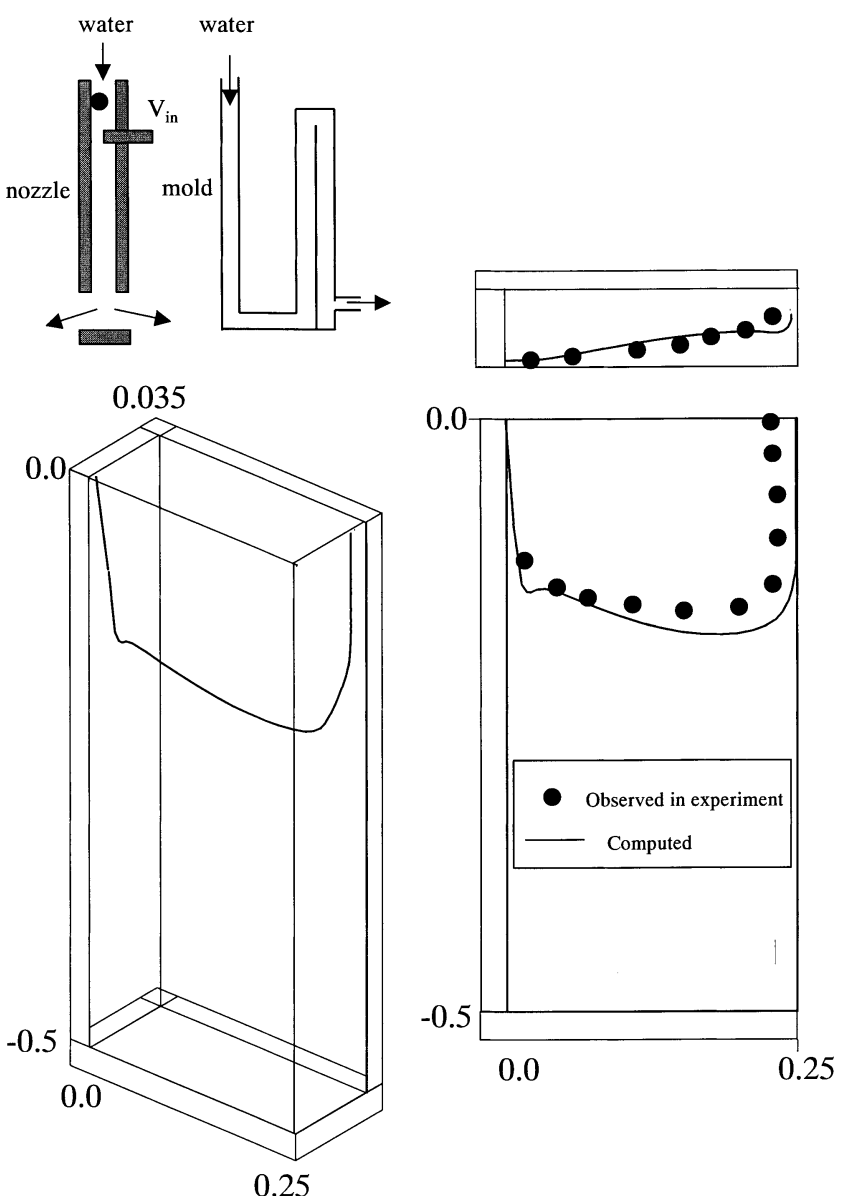

Fig. 2. Comparison of the observed trajectory in experiments with the numerical calculation in the water model. Diameter of sphere is $3 \mathrm{~mm}$, and density of sphere is $900 \mathrm{~kg} / \mathrm{m}^{3}$.
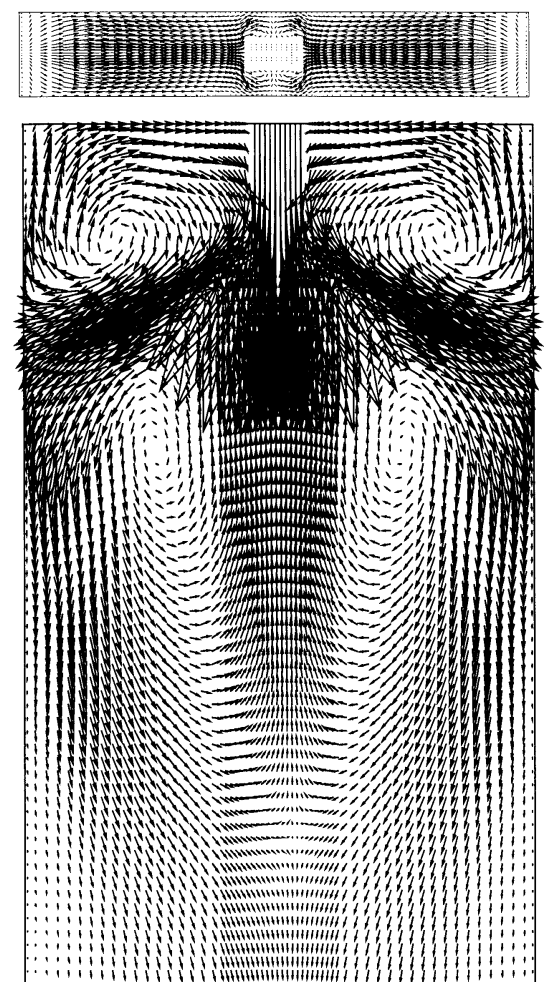

Fig. 3. Calculated flow field in the free surface and half thickness of slab in case that the nozzle is located at the center of mold. Maximum velocity at inlet of nozzle, $V_{\mathrm{in}},=1.75$ $\mathrm{m} / \mathrm{s}$.
Table 1. A quarter of the continuous casting strand of steel is chosen as the calculation domain, since twofold symmetry is assumed. Figure 4 illustrates the computed flow field with casting speed of $3.5 \mathrm{~m} / \mathrm{min}^{13)}$ These results are used to analyze the motion behavior of inclusion particles in the present and subsequent sections.

The distribution of inclusion particles in molten steel is assumed to be uniform; the initial locations are uniformly placed at nodes as shown in Fig. 5 within the horizontal section of the nozzle. It is necessary to obtain the lowest particle number to save the run time of computer. Figure 6

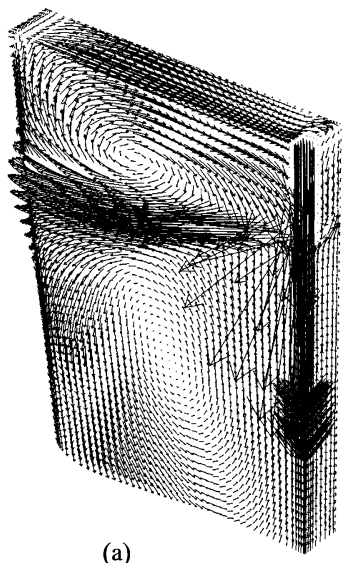

(a)

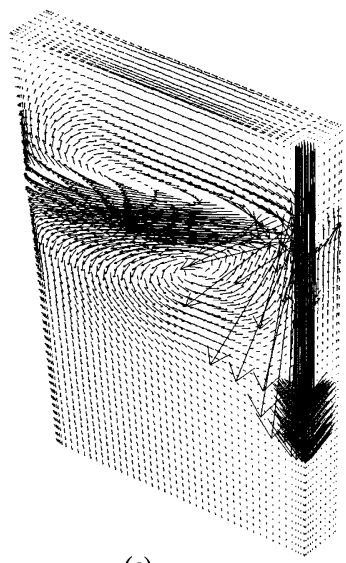

(c)

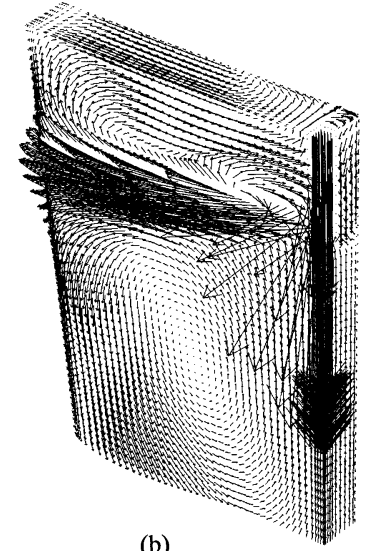

(b)

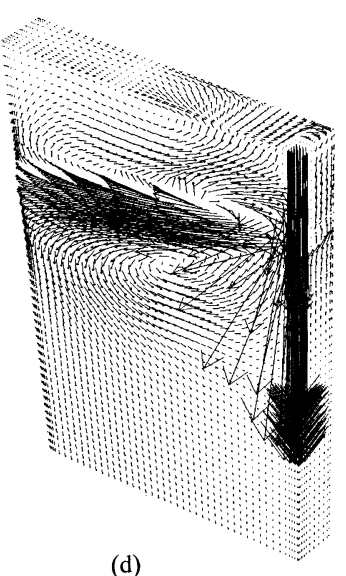

(d)
Fig. 4. The computed velocity distributions for three sections. Maximum velocity is $1.75 \mathrm{~m} / \mathrm{s}$. (a) without gas injection and magnetic field application, (b) with argon gas injection (bubbles size $3 \mathrm{~mm}, 10 \%$ gas at inlet), (c) with magnetic field application $\left(B_{\max }=0.315 \mathrm{~T}\right)$, and (d) with argon gas injection (bubbles size $3 \mathrm{~mm}, 10 \%$ gas at inlet) and magnetic field application $\left(B_{\max }=0.315 \mathrm{~T}\right)$.

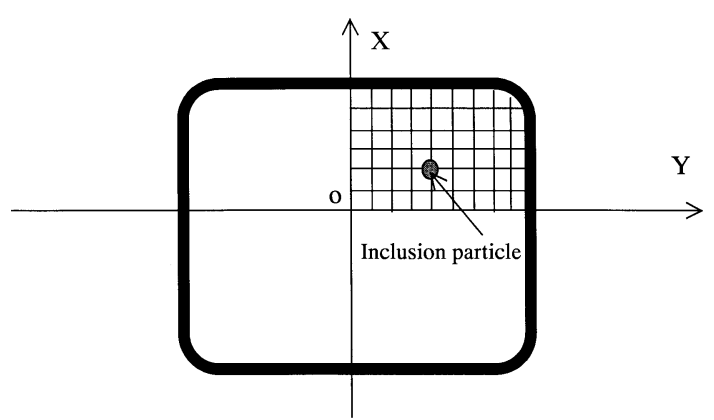

Fig. 5. Initial locations of inclusion particles at node point on section of the nozzle. 


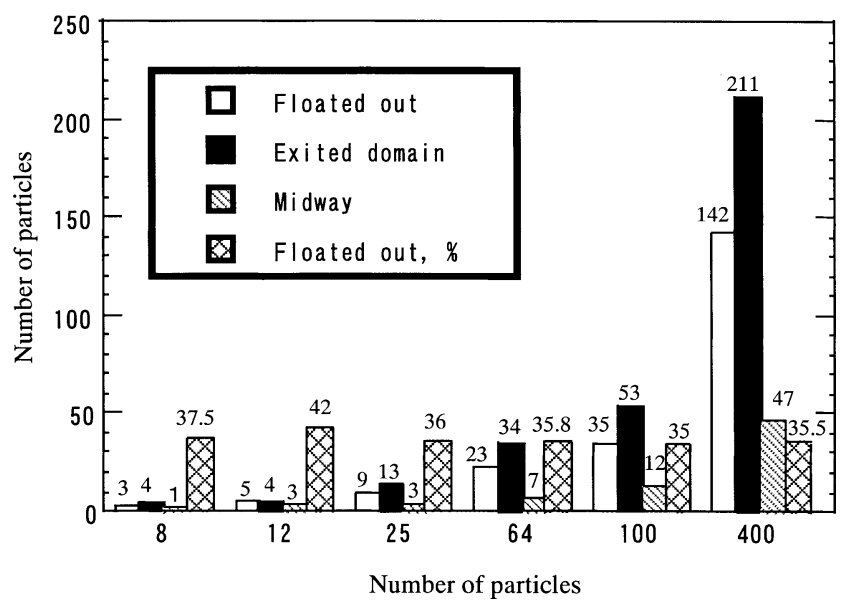

Fig. 6. Distribution of particles with the increase of number of inclusions. Diameter is $100 \mathrm{~mm}$.

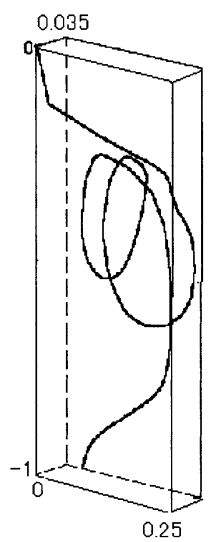

(a)

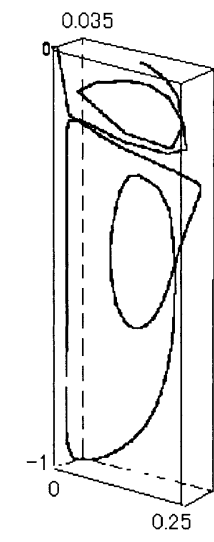

(b) shows the distribution of the particles with the increase of particle number. In "Midway", some inclusion particles always move in the recirculation zones within the tracing time. The effect of the number of particles on the rate of the floating out particles for the same flow field is included. It is observed that the rate is approximately constant, where the number of particles exceeds 60 under the present condition.

\subsection{Effects of the Argon Gas Injection and Static Magnetic Field Application}

The trajectory of a particle can be obtained by tracing the locations of a particle at different times. A single inclusion particle (diameter $100 \mu \mathrm{m}$ ) is traced for four cases with the same initial condition as shown in Fig. 7. The trajectories vary with the flow fields. When argon gas is not injected and magnetic field is not applied, the particle discharged from the nozzle enters the low recirculation zone, then experiences two spiral motions and finally exits the domain. The computed gas volume fractions without and with the
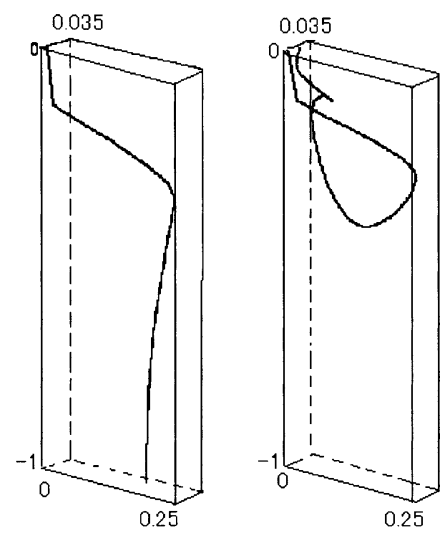

(c)

(d)

Fig. 7. Single particle trajectories with same initial condition. (a) Without gas injection and magnetic field application, (b) with argon gas injection (bubbles size $3 \mathrm{~mm}, 10 \%$ gas at inlet), (c) with magnetic field application $\left(B_{\max }=0.315 \mathrm{~T}\right)$, and $(\mathrm{d})$ with argon gas injection (bubbles size $3 \mathrm{~mm}, 10 \%$ gas at inlet) and magnetic field application $\left(B_{\max }=0.315 \mathrm{~T}\right)$.

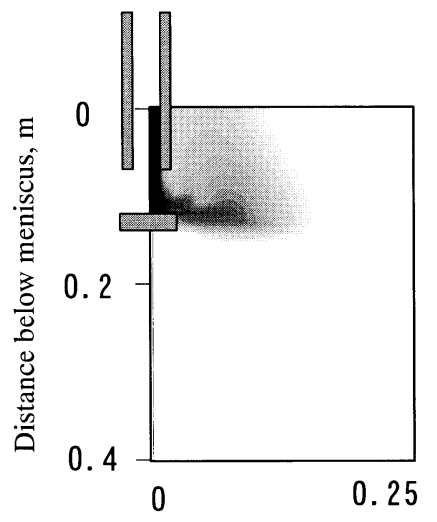

Distance from nozzle centerline, $\mathrm{m}$

(a)

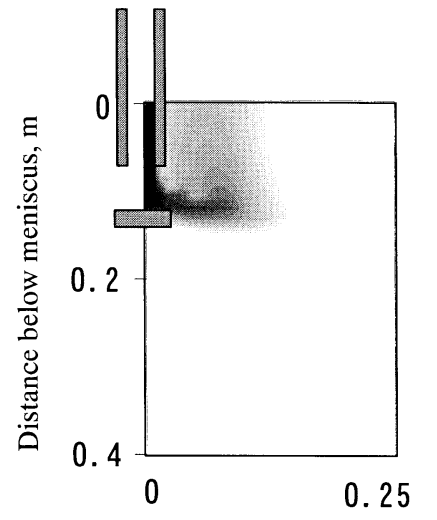

(b)

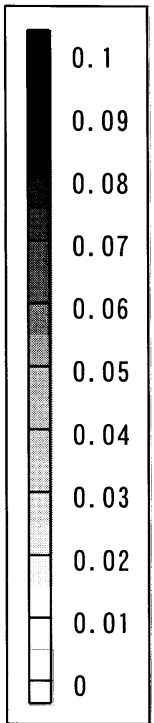

Fig. 8. The computed gas volume fraction at the thickness of slab. (a) Without and (b) with magnetic field application $\left(B_{\max }=0.315 \mathrm{~T}\right)$. 
effect of magnetic field are shown in Fig. 8. Argon gas injection changes the inclusion trajectory; the particle re-enters the upper recirculation zone after experiencing a spiral motion in the low recirculation zone, and finally floats out of the domain. When the magnetic field is applied, no spiral motion occurs and the particle slows down to exit the domain. The combination of argon gas injection and magnetic field application drives the inclusions to enter the active gas plume from the low velocity zone and improves the removal process of inclusions.

Figure 9 shows the velocities of inclusion particles with time. Horizontal straight lines indicate that the particle is removed from the calculation domain. The velocity peaks indicate that inclusions re-enter the jet zone in the flow field, and the magnitude of particle velocities is equivalent to the order of flow velocity of molten steel.

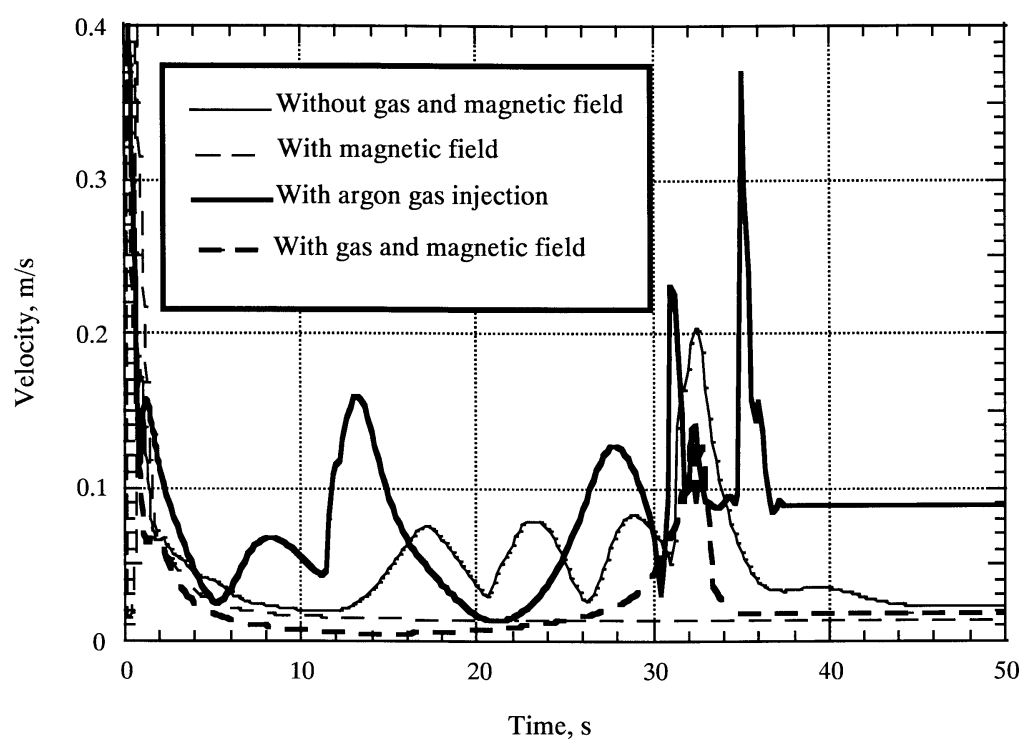

Fig. 9. Single particle velocities for four cases with same initial conditions.

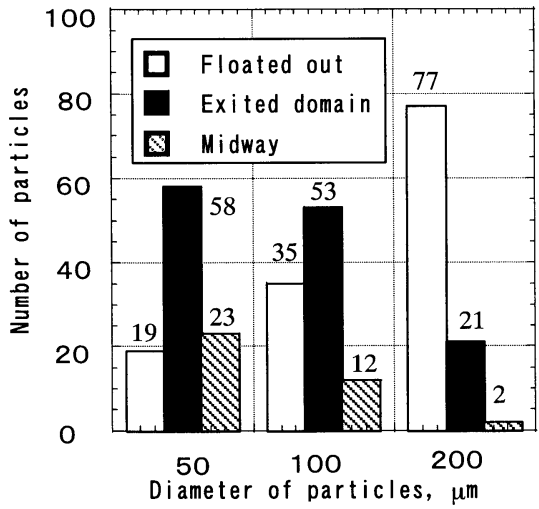

(a)

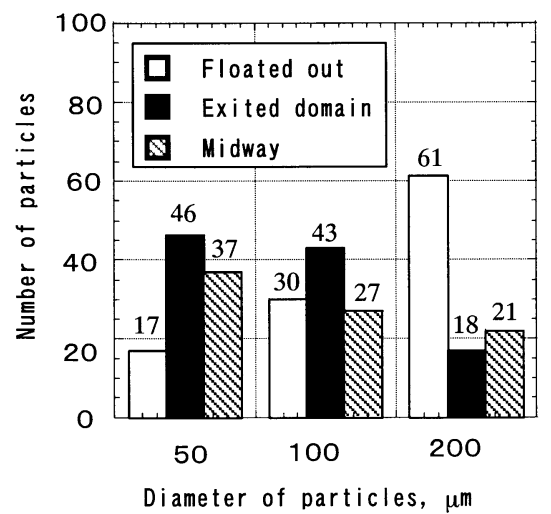

(c)

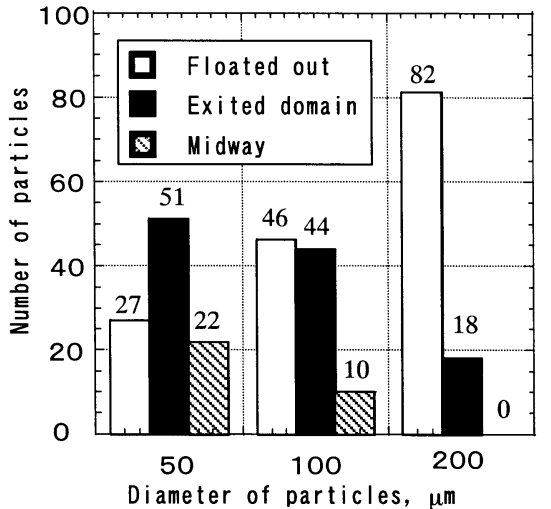

(b)

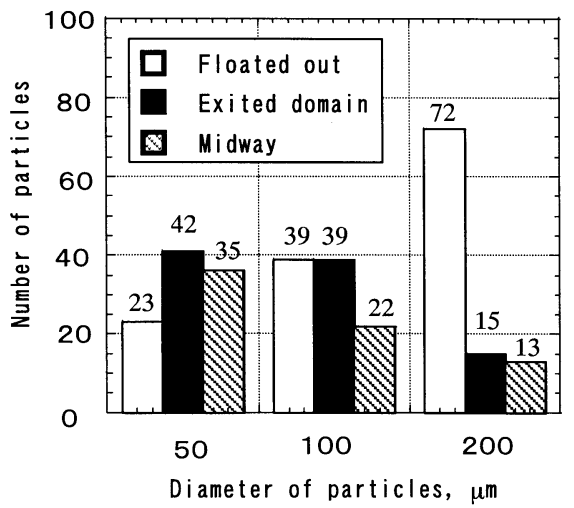

(d)

Fig. 10. Effect of particle diameter on the distribution of particles. (a) Without gas injection and magnetic field application, (b) with argon gas injection (bubbles size $3 \mathrm{~mm}, 10 \%$ gas at inlet), (c) with magnetic field application $\left(B_{\max }=0.315 \mathrm{~T}\right)$, and (d) with argon gas injection (bubbles size $3 \mathrm{~mm}, 10 \%$ gas at inlet) and magnetic field application $\left(B_{\max }=0.315 \mathrm{~T}\right)$. 
Under the conditions of 100 particles within the time period of $60 \mathrm{~s}$ at the casting speed of $3.5 \mathrm{~m} / \mathrm{min}$, the effect of particle size on the distribution of the particle is shown in Fig. 10. It is seen that the large-diameter inclusions easily float out to the surface, and the small-diameter inclusions also have the opportunity to float out. The effect of argon gas injection on the large diameter inclusion particles is observed as shown in Fig. 10 and numbers of inclusion particles in 'Midway' increase with the magnetic field application.

Figure 11 shows the trajectories of 64 particles (diameter $100 \mu \mathrm{m}$ ) within a period of $60 \mathrm{~s}$ for four cases. The result without argon gas injection and magnetic field application is shown in Fig. 11(a). The particles that enter the mold from the submerged entry nozzle are divided into two directions. Some particles move to the surface or upper recirculation zone, and the others enter the lower recirculation zone. However, some particles at the lower recirculation zone re-enter the jet zone of molten steel after the spiral movement, and then enter the upper recirculation zone or float up to the surface. At the same time, some particles in the upper recirculation zone also enter the lower recirculation zone. In the case of the argon gas injection, some parti-
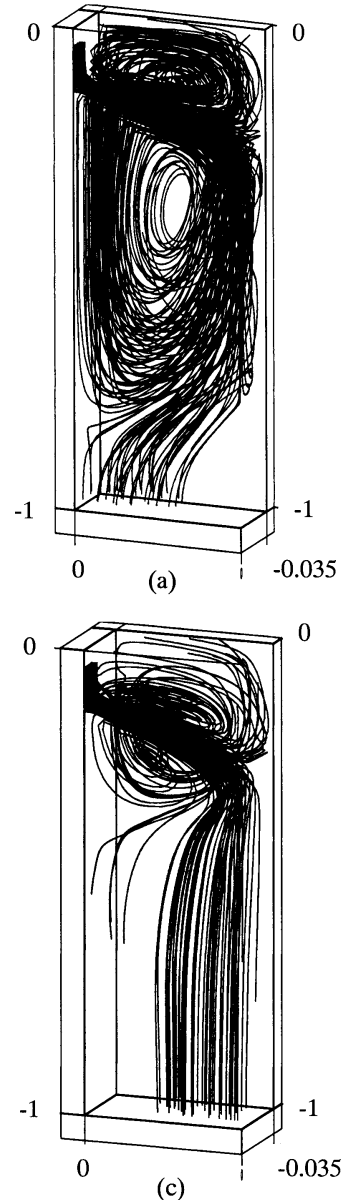

(c)
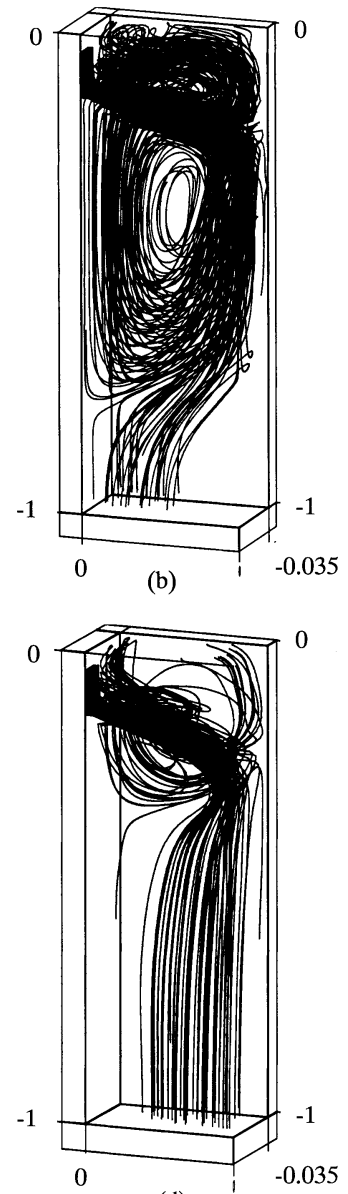

(d)
Fig. 11. Selected trajectories of 64 particles in the slab continuous casting strand. Diameter of particles is $100 \mathrm{~mm}$. (a) Without gas injection and magnetic field application, (b) with argon gas injection (bubbles size $3 \mathrm{~mm}, 10 \%$ gas at inlet), (c) with magnetic field application $\left(B_{\max }=\right.$ $0.315 \mathrm{~T}$ ), and (d) with argon gas injection (bubbles size $3 \mathrm{~mm}, 10 \%$ gas at inlet) and magnetic field application $\left(B_{\max }=0.315 \mathrm{~T}\right)$. cle trajectories become turbulent near the SEN in the upper recirculation zone shown in Figs. 11(b) and 11(d). This may be due to the fact that gas bubbles collect inclusion particles. The effect of magnetic field application on the trajectories of inclusion particles is shown in Figs. 11(c) and 11(d). The spiral movement disappears in both cases, and only a few particles move into the small recirculation zone. Most particles are divided into upward flow towards the surface and downward flow towards the exit of the slab.

Figure 12 illustrates the removal behavior of inclusion particles for four cases. One hundred particles were traced for $60 \mathrm{~s}$. Argon gas injection increases the removal rate of inclusion particles. On the other hand, magnetic field application decreases the rate of floating out of inclusion. This is why the upper recirculating flow is limited below the imposed DC field area and the inclusion loses a chance to the contact with molten steel surface. With the imposition of DC field at the lower part of the mold, the area of the lower recirculating flow also reduces to decreases the 'exited domain' number of inclusion and increase the 'Midway' number of inclusion.

\section{Conclusions}

A three-dimensional numerical simulation on the motion of inclusion particles in the continuous casting of slab was conducted to analyze the trajectories and velocities of inclusion particles considering the effects of argon gas injection and magnetic field application.

(1) The reliability of the numerical model is tested using the water model without the gas injection and magnetic field application. Reasonable agreement between computed and experimentally obtained sphere locations is observed and the slight difference is due to the rotation observed in the experiment. The dependence of particle distribution on the particle number is also examined. It is found that the rate of floating out is almost constant, where the number of particles exceeds 60 under the present condition.

(2) Without the argon gas injection and magnetic field application, inclusions are divided into two directions,

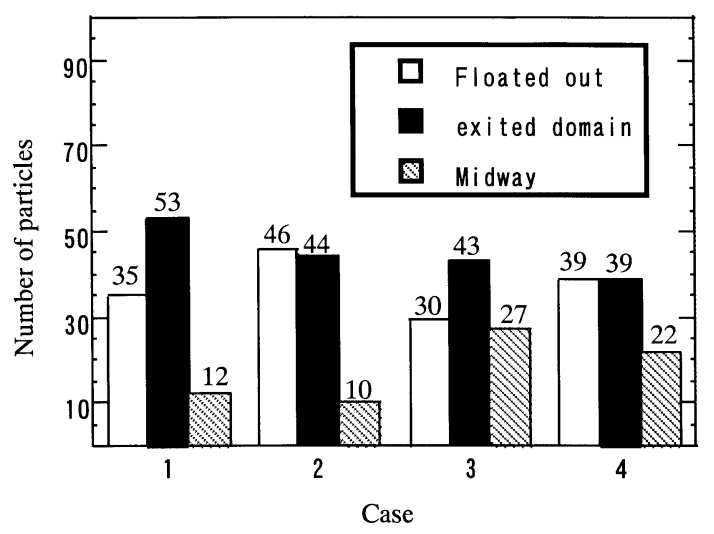

Fig. 12. Distribution of particles for four cases. Diameter of particles is $100 \mathrm{~mm}$ and number of particles is 100 . (1) Without gas injection and magnetic field application, (2) with argon gas injection (bubbles size $3 \mathrm{~mm}, 10 \%$ gas at inlet), (3) with magnetic field application $\left(B_{\max }=\right.$ $0.315 \mathrm{~T}$ ), and (4) with argon gas injection (bubbles size $3 \mathrm{~mm}, 10 \%$ gas at inlet) and magnetic field application $\left(B_{\max }=0.315 \mathrm{~T}\right)$. 
some particles move toward the surface or upper recirculation zone, and others enter the lower recirculation zone. Some particles re-enter the jet zone from the recirculation zones and then enter the opposite recirculation zone.

(3) When argon gas is injected, the trajectories of inclusion particles become turbulent in the gas plume due to the effect of gas bubbles. Argon gas injection increases the removal rate of inclusion particles.

(4) When the magnetic field is applied, the spiral trajectories of inclusion particles disappear and the particle velocities decrease significantly, and the rate of the floating out of inclusions decreases.

\section{Acknowledgments}

One of the authors, Baokuan Li, is grateful to the Natural Science Foundation of China for support of this research, Project No. 59604006 and No. 50274029.

\section{REFERENCES}

1) Y. H. Ho and W. S. Hwang: ISIJ Int., 36 (1996), No. 8, 1030.

2) B .G. Thomas and X. Huang: 76th Steelmaking Conf. Proc., ISSAIME, Warrendale, PA, (1993), 273.

3) B. G. Thomas, A. Denissv and H. Bai: 80th Steelmaking Conf. Proc., ISS-AIME, Warrendale, PA, (1997), 375.

4) S. Taniguchi, A.Kikuchi, T. Ise and N. Shoji: ISIJ Int., 36 (1996), Supplement, s117.
5) Y. Miki and B. G. Thomas: Metall. Mater. Trans. B, 30B (1999), No. $4,639$.

6) J. Nagai, K. Suzuki, S. Kojima and S. Kollbery: Iron Steel Eng., 61 (1984), No. 5, 41.

7) M. Zeze, H. Harada and E. Takeuchi: 76th Steelmaking Conf. Proc., ISS-AIME, Warrendale, PA, (1993), 267.

8) A. Idogawa, M. Sugizawa, S. Takeuchi, K. Sorimachi and T. Fujii: Mater. Sci. Eng., 173A (1993), 293.

9) A. Lehman, G. Tallback, S. Kollbery and H. Hackl: Proc. Int. Symp. on Electromagnetic Processing of Materials, ISIJ, Tokyo, (1994), 372.

10) K. H. Moon, H. K. Shin, B. J. Kim, J. Y. Chang, Y. S. Hwang and J. K. Yoon: ISIJ Int., 36 (1996), s201.

11) P. Gardin, J. F. Domgin, M. Anderhuber, J. M. Galpin, and J. Y. Lamant: The 3rd Int. Symp. on EPM, ISIJ, Tokyo, (2000), 422.

12) M. J. Cho, S. J. Kim, I. C. Kim, J. K. Kim, D. W. Cha and J. H. Park: The 3rd Int. Symp. on EPM, ISIJ, Tokyo, (2000), 176.

13) B. K. Li, T. Okane and T. Umeda: Metall. Mater. Trans. B, 31B (2000), No. 6, 1491

14) A. Sterl: J. Fluid Mech., 216 (1990), 161.

15) J. O. Hinze: Turbulence, McGraw-Hill Book Company, New York, (1975), 463.

16) R. Clift, J. R.Grace and M. E. Weber: Bubbles, Drop and Particles, Academic Press, New York, (1978).

17) P. Marty and A. Alemany: Metallurgical Application of Magnetohydrodynamics, Proc. of The UITAM Symp., ed. by H. K. Moffatt and M. R. E. Proctor, The Metal Society, London, (1984), 245.

18) B. G. Thomas, L. J. Mika and F. M. Najjar: Metall. Trans. B, 21B (1990), 387. 